\title{
Influence of interlayer stacking on gate-induced carrier accumulation in bilayer $\mathrm{MoS}_{2}$
}

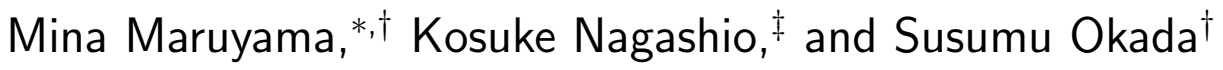 \\ $\dagger$ Department of Physics, Graduate School of Pure and Applied Sciences, University of \\ Tsukuba, 1-1-1 Tennodai, Tsukuba 305-8571, Japan \\ $\ddagger$ Department of Materials Engineering, Graduate School of Engineering, The University of \\ Tokyo, 7-3-1 Hongo, Tokyo 113-8656, Japan \\ E-mail: mmaruyama@comas.frsc.tsukuba.ac.jp \\ Phone: +81 (0)29 853 5921. Fax: +81 (0)29 8535924
}

\begin{abstract}
Atomic layered materials with a semiconducting electronic property have attracted much attention as a conducting channel in field effect transistors (FETs). Here, we investigate the electronic structures of bilayer $\mathrm{MoS}_{2}$ in a dual gate FET model by using first-principles total-energy calculations based on the density functional theory. Our calculations show that selective electron doping occurs in bilayer $\mathrm{MoS}_{2}$ under a perpendicular electric field owing to a band-offset between the positive and negative electrode sides of the $\mathrm{MoS}_{2}$ layers. Furthermore, we observe a further partial carrier distribution in bilayer $\mathrm{MoS}_{2}$ by decreasing the inter-layer interaction owing to a twisted stacking arrangement. It is expected that the positive-electrode-side layer works as a conducting channel, on the other hand, the negative-electrode-side layer works as a screening layer in bilayer $\mathrm{MoS}_{2}$-FET.
\end{abstract}

Keywords: $\mathrm{MoS}_{2}$, FET, electronic structure, electron doping, interlayer interaction, DFT 


\section{Introduction}

Transition metal dichalcogenides (TMDCs), such as $\mathrm{MoS}_{2}, \mathrm{MoSe}_{2}, \mathrm{WS}_{2}, \mathrm{WSe}_{2}$, and $\mathrm{MoTe}_{2}$, are possible forms of atomic layer materials. These consist of an atomic layer of transition metals that form a triangular lattice sandwiched by atomic layers of chalcogens arranged in a prismatic manner, to result in a hexagonal covalent network of these elements with a thickness of approximately $3 \AA .^{1-3}$ Owing to their chemical valence and hexagonal covalent networks, TMDCs possess chemically inert surfaces above and below their tight covalent network. Therefore, TMDCs are applicable as stable atomc layered materials and building blocks of van der Waals (vdW) heterostructures in which TMDCs are stacked on each other or with other two-dimensional materials, such as h-BN and graphene, through weak vdW interactions. $^{4}$

Most of the monolayer TMDCs are known to be semiconductors with a direct band gap at the $\mathrm{K}$ point, which strongly depends on the constituent elements. ${ }^{5}$ According to the semiconducting electronic structure, whose band gap is tunable by selecting an appropriate combination of transition metal and chalcogen, TMDCs are regarded as a constituent material of switching electronic devices, even though the carrier mobility is lower than that of graphene. ${ }^{6}$ Furthermore, because the versatile electronic structures of TMDCs depend on the constituent elements, the formation of vdW heterostructures causes exhibit further variation in their electronic structures that exceeds the simple sum of those of the constituent atom layer materials. Indeed, the electronic properties of vdW heterostructures of TMDCs are sensitive to the electronic band structures of the constituent layers and interlayer stacking arrangement. Most of the vdW heterostructures of TMDCs are Type II semiconductors. Their band gap is determined by the band edge offset of the constituent TMDCs. ${ }^{7-12}$ In contrast, the $\mathrm{MoS}_{2} / \mathrm{ReS}_{2}$ and $\mathrm{MoTe}_{2} / \mathrm{WSe}_{2}$ heterostructures are Type I semiconductors, ${ }^{13,14}$ owing to the large band edge offset between constituent layers.

For practical applications of TMDCs and their thin films to functional devices, the control of their electronic properties, by applying an external electric field that induces electron and 
hole doping, is crucial to the operation of such devices. ${ }^{15-18}$ The complex band edge alignment and the wave function distribution on such TMDC heterostructures cause an unusual carrier accumulation and depression in thin films by the gate electric field. ${ }^{19}$ In particular, precise control of the carrier density and distribution in TMDC thin films is highly demanded to endow them with new functionality and improve their device performance. Our previous first-principles calculations provided the possibility of free electron carriers outside $\mathrm{MoS}_{2}$ thin films ${ }^{19}$ and the asymmetric carrier accumulation in a $\mathrm{MoS}_{2} / \mathrm{WS}_{2}$ heterostructure, with respect to the TMDC arrangement at the electrode. ${ }^{20}$ However, it is still unclear how to control the accumulated carrier distribution in thin films.

In this work, we aim to theoretically investigate how to control the accumulated carrier distribution in a bilayer $\mathrm{MoS}_{2}$ field effect transistor (FET) by the external electric field, the doping concentration, and the interlayer stacking arrangement using the density functional theory (DFT). Our calculation showed that the carrier distribution on the bilayer $\mathrm{MoS}_{2}$ is tunable, whether the injected carrier is localized on one of the two layers or extended throughout the layers, by controlling the external electric field and electron density, which correspond to the control of the top and bottom gate voltages of a dual gate transistor. In addition to the electric field, the stacking misorientaiton of bilayer $\mathrm{MoS}_{2}$ enhances the carrier localization.

\section{Results and discussion}

We consider a dual gate FET of bilayer $\mathrm{MoS}_{2}$, in which the gate electrodes were simulated by a planar effective screening medium (ESM) with an infinite relative permittivity located above and below the bilayer $\mathrm{MoS}_{2}$ with a $0.60 \mathrm{~nm}$ vacuum spacing (Figure 1a). We controlled the electric field between the electrodes and the electron doping concentration in bilayer $\mathrm{MoS}_{2}$, instead of controlling the top and bottom gate voltages. As for the interlayer stacking arrangement of bilayer $\mathrm{MoS}_{2}$, we considered the $\mathrm{AB}$ and twisted (twisted angle $\theta=38^{\circ}$ ) 
arrangements to investigate the influence of interlayer stacking on the carrier accumulation and depression (Figure 1b, c). Note that the electronic structure of twisted $\mathrm{MoS}_{2}$ with the angle of $38^{\circ}$ is qualitatively the same as that with the $28^{\circ}$ (Figure S1). Thus, the bilayer $\mathrm{MoS}_{2}$ with the angle of $38^{\circ}$ may represent qualitative properties on the carrier accumulation associated with the inter layer stacking arrangement. The optimal interlayer spacing of bilayer $\mathrm{MoS}_{2}$ was 0.30 and $0.33 \mathrm{~nm}$ for the $\mathrm{AB}$ and twisted arrangements, respectively.

(a)

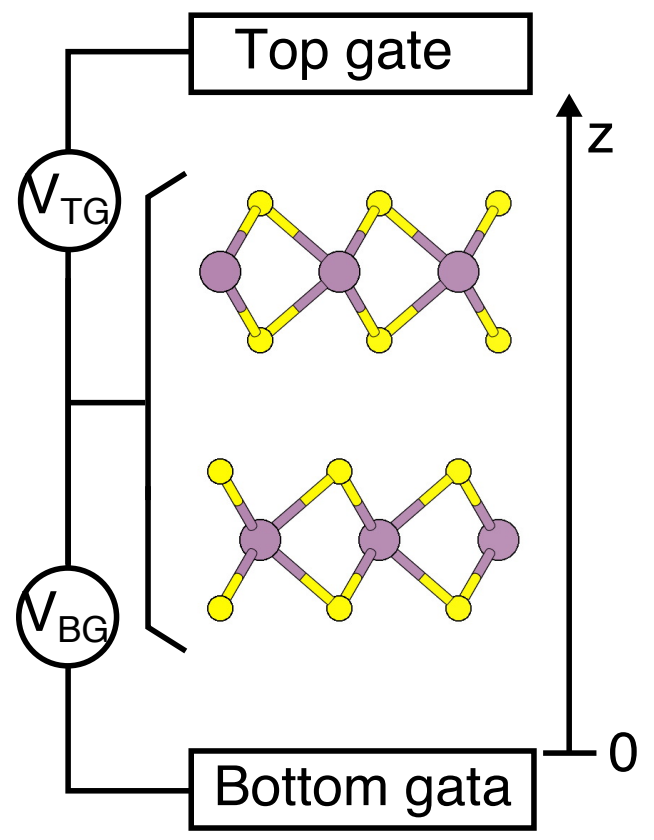

(b)

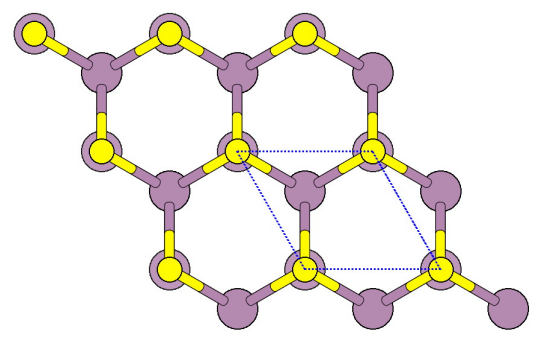

(c)

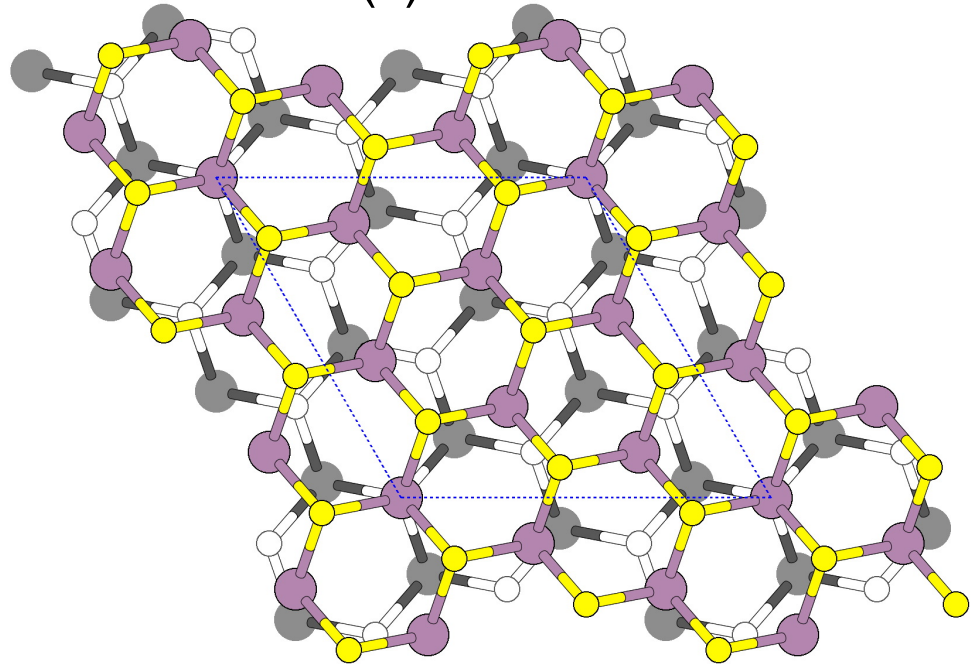

Figure 1: (a) Geometric structure of bilayer $\mathrm{MoS}_{2}$ in a dual-gate transistor structure. An electric field is applied along the $\mathrm{z}$ direction. Upper and lower rectangles denote the top and bottom gate electrodes, respectively. Purple and yellow balls denote the Mo and S atoms, respectively. Bilayer $\mathrm{MoS}_{2}$ with (b) $\mathrm{AB}$ and (c) twisted stacking structures. Purple and gray large balls indicate Mo atoms belonging to the top and bottom layers, respectively. Yellow and white small balls indicate $\mathrm{S}$ atoms belonging to the top and bottom layers, respectively.

Figure 2 shows the distribution of the electron accumulation and depression normal to the bilayer $\mathrm{MoS}_{2}$ with an $\mathrm{AB}$ and twisted interlayer arrangement under strong $(1.0 \mathrm{~V} / \mathrm{nm})$ 
and weak $(0.1 \mathrm{~V} / \mathrm{nm})$ electric fields and high $\left(1 \times 10^{14} / \mathrm{cm}^{2}\right)$ and low $\left(1 \times 10^{13} / \mathrm{cm}^{2}\right)$ electron doping. The electric field and carrier density considered here were relevant to those realized in experimental works. ${ }^{21,22}$ The accumulated carrier distribution was calculated by taking the difference between the valence charge density of bilayer $\mathrm{MoS}_{2}$ under an electric field and electron doping and that under an electric field without the excess electron. The distribution strongly depended on the electric field, doping concentration, and interlayer stacking arrangement. For the $\mathrm{AB} \mathrm{MoS}_{2}$ under a low doping concentration, the distribution of the electron accumulation weakly depended on the electric field. The electrons were extended throughout the layers under a low electric field (Figure 2a), while the density on the bottom layer was slightly higher than that on the top layer by $14 \%$ (Figure $2 \mathrm{~b}$ ). With an increase of the doping concentration, we observed the extended nature of the injected electron for both low and high electric fields (Figure 2c, d). For the bilayer $\mathrm{MoS}_{2}$ with a twisted arrangement, the asymmetric electron distribution was highly enhanced under the condition of a low doping concentration and a strong electric field. In this case, $85 \%$ of the electrons were accommodated in the bottom layer, although the system had structurally symmetric layers in terms of their constituent elements (Figure 2f). For the other external conditions, the injected electrons were distributed on both layers (Figure 2e, g, h). Therefore, one of the two layers of the bilayer TMDC dual gate FET with stacking misorientation acted as a screening layer against the other foreign materials, such as substrates and high-k dielectrics, for the electron doping under a strong electric field, even though the system solely consisted of a $\mathrm{MoS}_{2}$ layer. This characteristic feature was ascribed to the band edge misalignment between the top and bottom $\mathrm{MoS}_{2}$ and the weak interlayer coupling of the wavefunction between the layers in the twisted arrangement.

To confirm the interlayer wavefunction coupling, we investigated the electrostatic potential between the bottom and top gate electrodes for bilayer $\mathrm{MoS}_{2}$ with $\mathrm{AB}$ and twisted interlayer arrangements under a strong electric field (Figure 3). The electric field exhibits symmetric behavior with respect to the $\mathrm{MoS}_{2}$ layers: The electric field in the vacuum in 

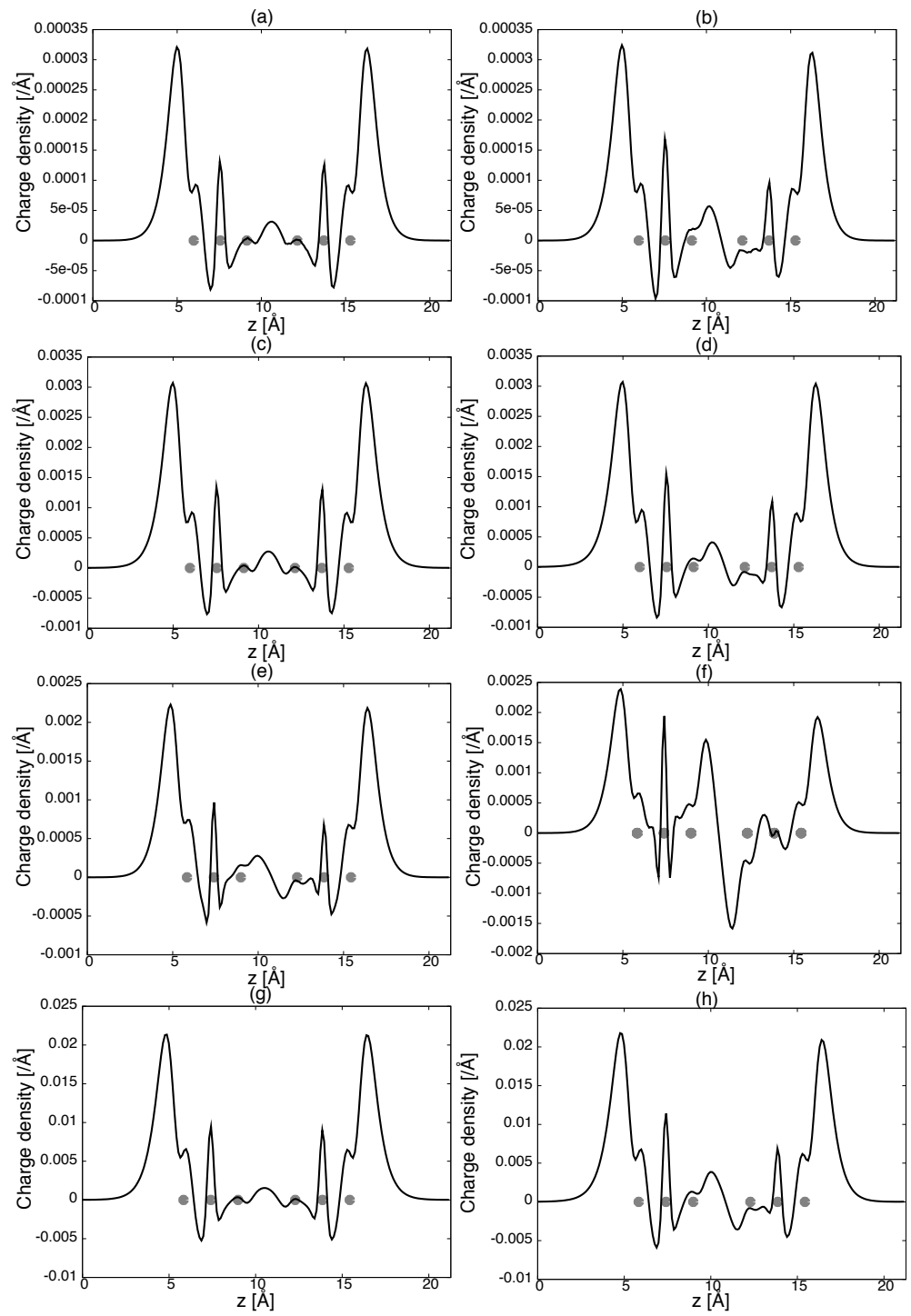

Figure 2: Plane averaged electron densities as a function of the $\mathrm{z}$ axis of the bilayer $\mathrm{MoS}_{2}$ with $\mathrm{AB}$ arrangement under (a) a low electric field and low doping concentration, (b) a high electric field and low doping concentration, (c) a low electric field and high doping concentration, and (d) a high electric field and high doping concentration, and those with twisted arrangement under (e) a low electric field and low doping concentration, (f) a high electric field and low doping concentration, (g) a low electric field and high doping concentration, and (h) a high electric field and high doping concentration. Gray circles denote the atomic position of $\mathrm{MoS}_{2}$. Positive and negative values correspond to the regions where electron accumulation and depression occur, respectively. 
front of the top and bottom gate electrodes are $F^{T}=F^{B}=1.9 \mathrm{~V} / \mathrm{nm}$, respectively. On the other hand, the field between $\mathrm{MoS}_{2}$ layers is obviously weaker than that in the outside vacuum region, indicating the interlayer stacking effect. By taking the ratio of the potential gradient at the interlayer region to the outside $\mathrm{MoS}_{2}$, we can estimate the screening rate at the interlayer spacing, which reflects the spilled charge associated with the polarization and interlayer hybridization of wavefunctions. The estimated rate was 0.46 and 0.67 for the $\mathrm{AB}$ and twisted arrangements, respectively, which indicated that the $\mathrm{MoS}_{2}$ layers in the twisted arrangement were electronically separated from each other compared with those in the AB arrangement. Thus, we can conclude that the band edge misalignment and the weak interlayer coupling caused highly asymmetric electron doping in a bilayer $\mathrm{MoS}_{2}$ dual-gate FET.

(a)

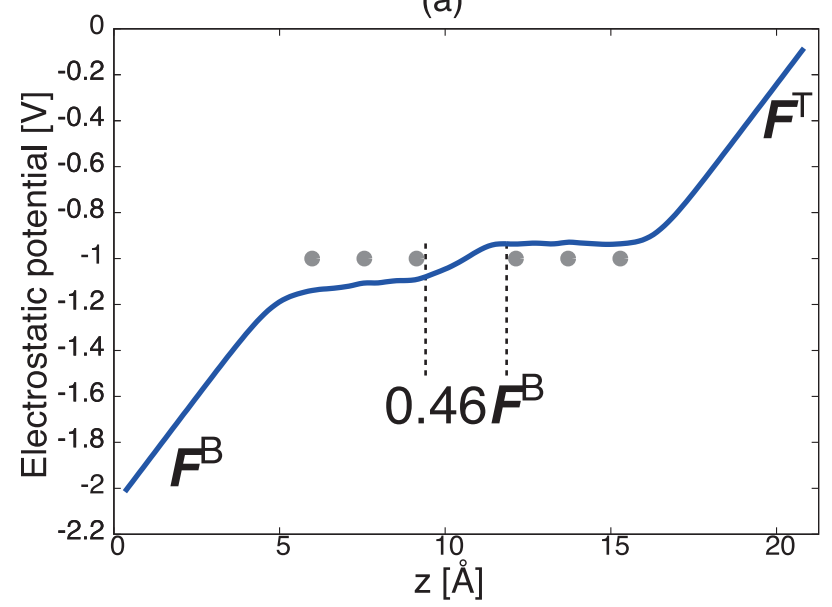

(b)

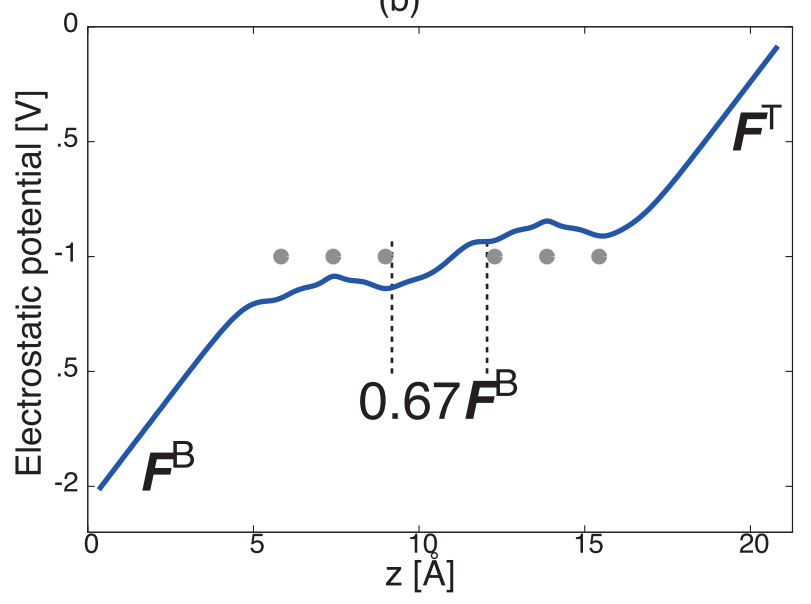

Figure 3: Electrostatic potential of bilayer $\mathrm{MoS}_{2}$ with (a) AB and (b) twisted arrangements along the z-axis. $F^{T}$ and $F^{B}$ indicate the electric field in vacuum region in front of the top and bottom gate electrodes, respectively. Gray circles correspond to the atomic position of $\mathrm{MoS}_{2}$.

Finally, it is worth predicting the strength of the electric field required to control the electron localization in bilayer $\mathrm{MoS}_{2}$ in dual-gate FETs. Figure 4a shows the two-dimensional map of the electron concentration in the top layer of bilayer $\mathrm{MoS}_{2}$ as functions of the external electric field and the electron concentration. The charge localization occurred under a low electron doping concentration and at a low electric field. However, the electron localization 
was sensitive to the increase of the electron density. The accommodated electron distribution immediately lost its localized nature with the increase of the doping concentration. The asymmetric and symmetric electron distribution associated with the field and the doping concentration was interpreted by the cartoon in Figure 4b. For the low electric field region, the band edge misalignment between the layer was too small to cause electron localization on one of the two layers, while the doped carriers were accommodated in both layers. For a strong electric field, the injected carriers exhibited an asymmetric distribution under a low doping concentration. In contrast, under a high doping concentration, the carriers were extended through the layers, because a large number of carriers were accommodated not only at the lower conduction band edge but also at the higher conduction band edge.

\section{Conclusion}

Based on DFT with the ESM method, we investigated the electronic properties of bilayer $\mathrm{MoS}_{2}$ under an external electric field with excess electrons to provide theoretical insight into the microscopic mechanism of carrier accumulation in a $\mathrm{MoS}_{2}$ dual-gate FET. Our calculations showed an asymmetric carrier distribution in bilayer $\mathrm{MoS}_{2}$ with a twisted interlayer structure under a strong electric field and low doping concentration, because of the band edge misalignment owing to the electric field and the weak interlayer wavefunction coupling. The asymmetric electron distribution in bilayer $\mathrm{MoS}_{2}$ with a twisted structure is robust against an external electric field under a low doping concentration, while it is sensitive to an increase of the doping concentration. Based on these results, we propose that the bilayer TMDCs with stacking misorientation could be applicable as a stable conducting channel of a dual gate transistor with high carrier mobility, in which one of the two layers protects the channel from the external disturbances caused by the substrates and dielectrics. 
(a)

Electric field [V/nm]

\begin{tabular}{|c|c|c|c|c|c|c|c|c|c|c|c|}
\hline \multirow{3}{*}{$\frac{E}{0}$} & & 0.1 & 0.2 & 0.3 & 0.4 & 0.5 & 0.6 & 0.7 & 0.8 & 0.9 & 1.0 \\
\hline & 1 & 44.1 & 37.3 & 31.3 & 25.7 & 20.9 & 17.3 & 16.9 & 16.5 & 15.8 & B 15.4 \\
\hline & 2 & 46.4 & 43.5 & 39.9 & 36.9 & $|33.4|$ & 31.7 & 29.5 & 26.6 & 24.8 & 323. \\
\hline & 3 & 47.2 & 44.9 & 42.9 & 40.5 & 38.4 & 37.2 & 35.3 & 33.1 & 31.0 & 29.4 \\
\hline & 4 & 47.7 & 45.9 & 44.4 & 42.5 & 40.6 & 39.8 & 38.2 & 36.8 & 34.8 & \begin{tabular}{l|l|l}
3 & 33.3
\end{tabular} \\
\hline & 5 & 7.8 & 4 & 4 & 43.6 & 42.1 & 41.5 & 40.2 & 3.9 & 37. & $\begin{array}{l}3 \\
35\end{array}$ \\
\hline & 6 & 7. & 6.8 & 45.8 & 4.5 & 43.3 & 42.8 & 41.6 & 40.4 & 39.1 & 37.7 \\
\hline & 7 & 48.0 & 47.1 & 46.2 & 45.1 & 44.1 & 43.5 & 42.5 & 41.5 & 40.3 & \begin{tabular}{|l|l|l} 
& 39.1 \\
\end{tabular} \\
\hline & 8 & 48.1 & 47.3 & 46.6 & 45.5 & 44.6 & 4 & 41.6 & 42.3 & 41.2 & \begin{tabular}{|l|l}
2 & 40.2
\end{tabular} \\
\hline & 9 & 48.2 & 1474 & 46.7 & 45.8 & 45.1 & $1 \Delta 46$ & 8 & 42.9 & 42 & 1 \\
\hline & & & & & & & & 4 & ? & 42.6 & $\begin{array}{l}6 \\
6\end{array}$ \\
\hline
\end{tabular}

\begin{tabular}{|l}
$\boldsymbol{F}_{\mathrm{s}}$ \\
\hline+ \\
$\frac{1}{8}$ \\
$\frac{\mathbf{y}}{\mathrm{c}}$ \\
$\frac{\mathrm{d}}{\Phi}$ \\
\hline
\end{tabular}

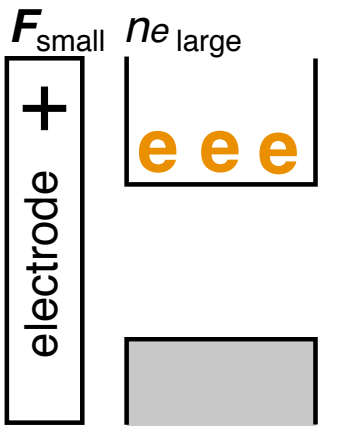

(b)
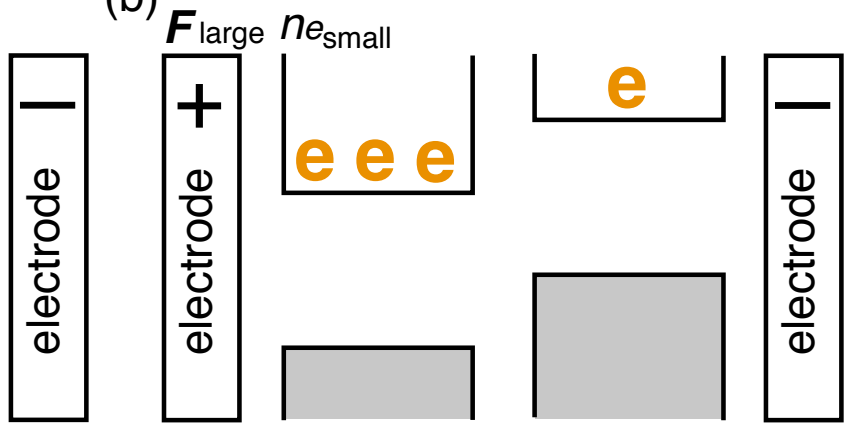

$\boldsymbol{F}_{\text {large }} \boldsymbol{n e}_{\text {large }}$

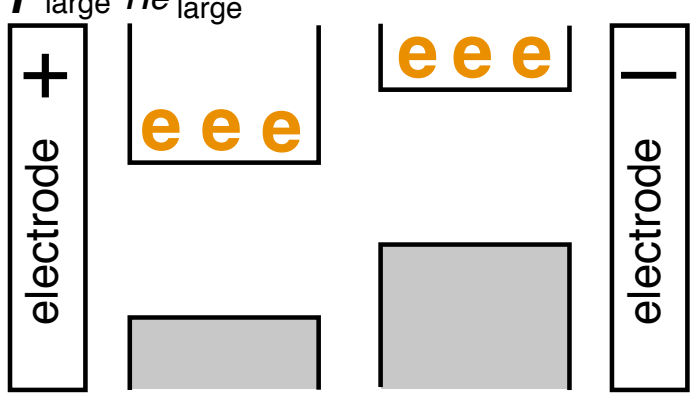

Figure 4: (a) Two-dimensional plot of the electron concentration of the top layer of bilayer $\mathrm{MoS}_{2}$ under an electric field with excess electrons. Unit of the concentration is \%. (b) Schematic views of the band edge alignment of bilayer $\mathrm{MoS}_{2}$ under a field with excess electrons. 


\section{Methods}

All calculations were based on $\mathrm{DFT}^{23,24}$ as implemented in the program package Simulation Tool for Atom TEchnology (STATE). ${ }^{25}$ The exchange-correlation potential energy among interacting electrons is described using the generalized gradient approximation with the Perdew-Burke-Ernzerhof functional. ${ }^{26,27}$ The weak dispersive interaction between $\mathrm{MoS}_{2}$ layers was treated by adopting the vdW-DF2 with the C09 exchange-correlation functional. ${ }^{28,29}$ It should be noted that the dispersion force in chalcogenides with structural disorders are sensitive to the choice of the functional form. ${ }^{30}$ We used ultrasoft pseudopotential for describing electrons-nuclei interations. ${ }^{31}$ Parameters associated with the cutoff energies of valence wave function and the force criteria are found in elsewhere. ${ }^{20}$ The external electric field and excess carriers on bilayer $\mathrm{MoS}_{2}$ are treated using the ESM method ${ }^{32,33}$ (see supporting information S2). During the electronic structure calculations under an electric field, the geometric structures of the bilayer $\mathrm{MoS}_{2}$ were fixed to their optimized structures obtained under zero electric field and neutral condition. The maximum force under the highest electric filed with the highest carrier concentration is $2.9 \times 10^{-3} \mathrm{HR} / \mathrm{au}$ which is too small to cause substantial structural modulation.

\section{Acknowledgement}

The authors thank JST-CREST Grant Numbers JPMJCR1532 and JPMJCR1715 from the Japan Science and Technology Agency, JSPS KAKENHI Grant Numbers JP17H01069, JP16H00898, and JP16H06331 from the Japan Society for the Promotion of Science, the Joint Research Program on Zero-Emission Energy Research, Institute of Advanced Energy, Kyoto University, and University of Tsukuba Basic Research Support Program Type S. Part of the calculations was performed on an NEC SX-Ace at the Cybermedia Center at Osaka University and on an SGI ICE XA/UV at the Institute of Solid State Physics, The University of Tokyo. 


\section{References}

(1) Friend R. H.; Yoffe, A. D. Electronic Properties of Intercalation Complexes of the Transition Metal Dichalcogenides. Adv. Phys. 1987, 36, 1-94.

(2) Wang, Q. H.; Kalantar-Zadeh, K.; Kis, A.; Coleman, J. N.; Strano, M. S. Electronics and Optoelectronics of Two-Dimensional Transition Metal Dichalcogenides. Nat. Nanotechnol. 2012, 7, 699-712.

(3) Mak, K. F.; Lee, C.; Hone, J.; Shan, J.; Heinz, T. F. Atomically Thin MoS 2 : A New Direct-Gap Semiconductor. Phys. Rev. Lett. 2010, 105, 136805.

(4) Lee, G-H.; Yu, Y.-J.; Cui, X.; Petrone, N.; Lee, C.-H.; Choi, M. S.; Lee, D.-Y.; Lee, C.; Yoo, W. J.; Watanabe, K.; Taniguchi, T.; Nuckolls, C.; Kim, P.; Hone, J. Flexible and Transparent $\mathrm{MoS}_{2}$ Field-Effect Transistors on Hexagonal Boron Nitride-Graphene Heterostructures. ACS Nano 2013, 7, 7931-7936.

(5) Splendiani, A .; Sun, L.; Zhang, Y.; Li, T.; Kim, J.; Chim, C.-Y.; Galli, G.; Wang, F. Emerging Photoluminescence in Monolayer $\mathrm{MoS}_{2}$. Nano Lett. 2010, 10, 1271-1275.

(6) Radisavljevic, B.; Radenovic, A.; Brivio, J.; Giacometti, V.; Kis, A. Single-Layer MoS 2 Transistors. Nat. Nanotechnol. 2011, 6, 147-150.

(7) Lee, C.-H.; Lee, G.-H.; van der Zande, A. M.; Chen, W.; Li, Y.; Han, M.; Cui, X.; Arefe, G.; Nuckolls, C.; Heinz, T. F.; Guo, J.; Hone, J.; Kim, P. Atomically Thin p-n Junctions with Van der Waals Heterointerfaces. Nat. Nanotech. 2014, 9, 676-681.

(8) Rivera, P.; Schaibley, J. R.; Jones, A. M.; Ross, J. S.; Wu, S.; Aivazian, G.; Klement, P.; Seyler, K.; Clark, G.; Ghimire, N. J.; Yan, J.; Mandrus, D. G.; Yao, W.; Xu, X. Observation of Long-Lived Interlayer Excitons in Monolayer $\mathrm{MoSe}_{2}-\mathrm{WSe}_{2}$ Heterostructures. Nat. Commun. 2015, 6, 6242. 
(9) Zhang, K.; Zhang, T.; Cheng, G.; Li, T.; Wang, S.; Wei, W.; Zhou, X.; Yu, W.; Sun, Y.; Wang, P.; Zhang, D.; Zeng, C.; Wang, X.; Hu, W.; Fan, H. J.; Shen, G.; Chen, X.; Duan, X.; Chang, K.; Dai, N. Interlayer Transition and Infrared Photodetection in Atomically Thin Type-II MoTe $2 / \mathrm{MoS}_{2}$ Van der Waals Heterostructures. ACS Nano 2016, 10, 3852-3858.

(10) Wang, K.; Huang, B.; Tian, M.; Ceballos, F.; Lin, M.-W.; Mahjouri-Samani, M.; Boulesbaa, A.; Puretzky, A. A.; Rouleau, C.; Yoon, M.; Zhao, H.; Xiao, K.; Duscher, G.; Geohegan, D. B. Interlayer Coupling in Twisted $\mathrm{WSe}_{2} / \mathrm{WS}_{2}$ Bilayer Heterostructures Revealed by Optical Spectroscopy. ACS Nano 2016, 10, 6612-6622.

(11) Kozawa, D.; Carvalho, A.; Verzhbitskiy, I.; Giustiniano, F.; Miyauchi, Y.; Mouri, S.; Castro Neto, A. H.; Matsuda, K.; Eda, G. Evidence for Fast Interlayer Energy Transfer in $\mathrm{MoSe}_{2} / \mathrm{WS}_{2}$ Heterostructures. Nano Lett. 2016, 16, 4087-4093.

(12) Mouri, S.; Zhang, W.; Kozawa, D.; Miyauchi, Y.; Eda, G.; Matsuda, K. Thermal Dissociation of Inter-Layer Excitons in $\mathrm{MoS}_{2} / \mathrm{MoSe}_{2}$ Hetero-Bilayers. Nanoscale 2017, 9, 6674-6679.

(13) Bellus, M. Z.; Li, M.; Lane, S. D.; Ceballos, F.; Cui, Q.; Zeng, X. C.; Zhao, H. Type-I Van der Waals Heterostructure Formed by $\mathrm{MoS}_{2}$ and $\mathrm{ReS}_{2}$ Monolayers. Nanoscale Horiz. 2017, 2, 31-36.

(14) Yamaoka, T.; Lim, H. E.; Koirala, S.; Shinokita, K.; Maruyama, M.; Okada, S.; Miyauchi, Y.; Matsuda, K. Efficient Photocarrier Transfer and Effective Photoluminescence Enhancement in Type I Monolayer $\mathrm{MoTe}_{2} / \mathrm{WSe}_{2}$ Heterostructure. Adv. Funct. Mater. 2018, 28, 1801021.

(15) Zhang, Y.; Ye, J.; Matsuhashi, Y.; Iwasa, Y. Ambipolar $\mathrm{MoS}_{2}$ Thin Flake Transistors. Nano Lett. 2012, 12, 1136-1140. 
(16) Pu, J.; Yomogida, Y.; Liu, K. K.; Li, L. J.; Iwasa, Y.; Takenobu, T. Highly Flexible $\mathrm{MoS}_{2}$ Thin-Film Transistors with Ion Gel Dielectrics. Nano Lett. 2012, 12, 4013-4017.

(17) Xia, P.; Feng, X.; Ng, R. J.; Wang, S.; Chi, D.; Li, C.; He, Z.; Liu, X.; Ang, K.-W. Impact and Origin of Interface States in MOS Capacitor with Monolayer $\mathrm{MoS}_{2}$ and $\mathrm{HfO}_{2}$ High- $k$ Dielectric. Sci. Rep. 2017, 7, 40669.

(18) Siao, M. D.; Shen, W. C.; Chen, R. S.; Chang, Z. W.; Shih, M. C.; Chiu, Y. P.; Cheng, C.-M. Two-Dimensional Electronic Transport and Surface Electron Accumulation in $\mathrm{MoS}_{2}$. Nat. Commun. 2018, 9, 1442.

(19) Cuong, N. T.; Otani, M.; Okada, S. Gate-Induced Electron-State Tuning of $\mathrm{MoS}_{2}$ : First-Principles Calculations. J. Phys.: Condens. Matter 2014, 26, 135001.

(20) Maruyama, M.; Okada, S. Asymmetric Carrier Accumulation in Van der Waals Heterostructures of $\mathrm{MoS}_{2} / \mathrm{WS}_{2}$ under an External Electric Field Appl. Phys. Express 2019, 12,075008 .

(21) Chu, T.; Ilatikhameneh, H.; Klimeck, G.; Rahman, R.; Chen, Z. Electrically Tunable Bandgaps in Bilayer $\mathrm{MoS}_{2}$. Nano Lett. 2015, 15, 8000-8007.

(22) Yuan, H.; Shimotani, H.; Ye, J.; Yoon, S.; Allah, H.; Tsukazaki, A.; Kawasaki, M.; Iwasa, Y. Electrostatic and Electrochemical Nature of Liquid-Gated Electric-DoubleLayer Transistors Based on Oxide Semiconductors. J. Am. Chem. Soc. 2019, 132, 1840218407.

(23) Hohenberg, P.; Kohn, W. Inhomogeneous Electron Gas. Phys. Rev. 1964, 136, B864B871.

(24) Kohn, W.; Sham, L. J. Self-Consistent Equations including Exchange and Correlation Effects. Phys. Rev. 1965, 140, A1133-A1138. 
(25) Morikawa, Y.; Iwata, K.; Terakura, K. Theoretical Study of Hydrogenation Process of Formate on Clean and Zn Deposited Cu(1 11 1) Surfaces. Appl. Surf. Sci. 2001, 169-170, $11-15$.

(26) Perdew, J. P.; Burke, K.; Ernzerhof, M. Generalized Gradient Approximation Made Simple. Phys. Rev. Lett. 1996, 77, 3865-3868.

(27) Perdew, J. P.; Burke, K.; Ernzerhof, M. Generalized Gradient Approximation Made Simple [Phys. Rev. Lett. 77, 3865 (1996)]. Phys. Rev. Lett. 1997, 78, 1396.

(28) Lee, K.; Murray, É. D.; Kong, L.; Lundqvist, B. I.; Langreth, D. C. Higher-Accuracy Van der Waals Density Functional. Phys. Rev. B 2010, 82, 081101(R).

(29) Cooper, V. R. Van der Waals Density Functional: An Appropriate Exchange Functional. Phys. Rev. B 2010, 81, 161104(R).

(30) Massobrio, C.; Martin, E.; Chaker, Z.; Boero, M.; Bouzid, A.; Roux, S. L.; Ori, G. Sensitivity to Dispersion Forces in First-Principles Modeling of Disordered Chalcogenides. Front. Mater. 2018, 5, 78.

(31) Vanderbilt, D. Soft Self-Consistent Pseudopotentials in a Generalized Eigenvalue Formalism. Phys. Rev. B 1990, 41, 7892-7895.

(32) Otani, M.; Sugino, O. First-Principles Calculations of Charged Surfaces and Interfaces: A Plane-Wave Nonrepeated Slab Approach. Phys. Rev. B 2006, 73, 115407.

(33) Maruyama, M.; Okada, S. Energetics and Electronic Structure of Triangular Hexagonal Boron Nitride Nanoflakes. Sci. Rep. 2018, 8, 16657. 


\section{Graphical TOC Entry}

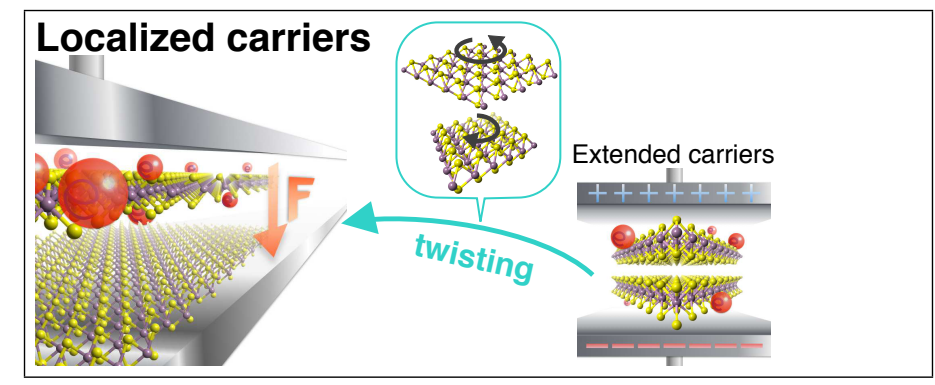

